\title{
Long-Term Disease Stability Assessed by the Expanded Disability Status Scale in Patients Treated with Cladribine Tablets $3.5 \mathrm{mg} / \mathrm{kg}$ for Relapsing Multiple Sclerosis: An Exploratory Post Hoc Analysis of the CLARITY and CLARITY Extension Studies
}

\author{
Gavin Giovannoni (D) - Giancarlo Comi (D) Kottil Rammohan (D) Peter Rieckmann • \\ Fernando Dangond (D) · Birgit Keller (D) - Dominic Jack (D) P Patrick Vermersch (D)
}

Received: April 7, 2021 / Accepted: July 16, 2021 / Published online: August 9, 2021

(C) The Author(s) 2021

\section{ABSTRACT}

Introduction: In the Cladribine Tablets Treating Multiple Sclerosis Orally (CLARITY) study, cladribine tablets significantly reduced relapse

G. Giovannoni $(\bowtie)$

Blizard Institute, Barts and The London School of Medicine and Dentistry, Queen Mary University of London, 4 Newark St, Whitechapel, London E1 2AT, UK

e-mail: g.giovannoni@qmul.ac.uk

G. Comi

Casa di Cura Privata del Policlinico, Università Vita-

Salute San Raffaele, Milan, Italy

K. Rammohan

MS Research Center, University of Miami School of Medicine, Miami, FL, USA

P. Rieckmann

Department of Neurology, Medical Park Loipl and

University of Erlangen, Erlangen, Germany

F. Dangond

EMD Serono Research and Development Institute, Inc., Billerica, MA, USA

B. Keller

The healthcare business of Merck KGaA, Darmstadt, Germany

D. Jack

Merck Serono Ltd (an affiliate of Merck KGaA,

Darmstadt, Germany), Feltham, UK

P. Vermersch

Univ. Lille, Inserm U1172 LilNCog, CHU Lille, FHU

Precise, Lille, France rates and improved findings on magnetic resonance imaging versus placebo in patients with relapsing multiple sclerosis. In the CLARITY Extension study, treatment with cladribine tablets for 2 years followed by placebo for 2 years produced similar clinical benefits to 4 years of cladribine tablets. The objective of this exploratory post hoc analysis was to evaluate long-term disease stability (assessed by the Expanded Disability Status Scale [EDSS] score) after treatment with cladribine tablets.

Methods: Patients enrolled into CLARITY Extension who were previously randomized to cladribine tablets $3.5 \mathrm{mg} / \mathrm{kg}$ in the CLARITY study were included in this post hoc analysis. Two treatment groups were investigated-patients randomized to cladribine tablets $3.5 \mathrm{mg} /$ $\mathrm{kg}$ in CLARITY and thereafter randomized to placebo in CLARITY Extension (the CP3.5 group) or to cladribine tablets $3.5 \mathrm{mg} / \mathrm{kg}$ in CLARITY Extension (the CC7 group). In each treatment group, EDSS scores at 6-month intervals, EDSS score improvement/worsening each year, and time to 3- and 6-month confirmed EDSS progression were assessed from CLARITY baseline over 5 years of follow-up (including a variable bridging interval between studies). All analyses are descriptive, and no statistical comparisons were performed for between-treatment group differences.

Results: The median (95\% confidence interval [CI]) EDSS score for patients in the CP3.5 group at 5 years was $2.5(2.0-3.5)$ compared with 3.0 
(2.5-3.5) at baseline. In the CC7 group, median EDSS score $(95 \% \mathrm{CI})$ at 5 years was 2.0 (2.0-3.0) compared with 2.5 (2.5-3.0) at baseline. During year 5 for the CP3.5 group, and based on changes in minimum score each year, EDSS score stability was observed in $53.9 \%$ of patients, improvement in $21.3 \%$, and worsening in $24.7 \%$. In the CC7 group, EDSS score remained stable in $66.1 \%$, improved in $18.1 \%$, and worsened in $15.8 \%$ of patients. Over $70 \%$ of patients in both treatment groups did not show 3- or 6-month confirmed EDSS progression at 5 years from CLARITY baseline.

Conclusions: These findings confirm long-term beneficial effects on disability afforded by either the recommended dose of cladribine tablets over 4 years (cumulative dose, $3.5 \mathrm{mg} / \mathrm{kg}$ ) or a higher cumulative dose.

Trial Registration: ClinicalTrials.gov NCT0021 3135 (CLARITY); NCT00641537 (CLARITY Extension).

Keywords: Cladribine tablets; Multiple sclerosis; Disease stability; CLARITY; CLARITY Extension

\section{Key Summary Points}

This exploratory post hoc analysis evaluated long-term disease stability (assessed by the Expanded Disability Status Scale [EDSS] score) among patients with relapsing multiple sclerosis who were treated with cladribine tablets in the Cladribine Tablets Treating Multiple Sclerosis Orally (CLARITY) and CLARITY Extension studies.

The study population comprised patients randomized to cladribine tablets $3.5 \mathrm{mg} /$ $\mathrm{kg}$ in CLARITY and thereafter, in CLARITY Extension, randomized to placebo (the CP3.5 group) or to cladribine tablets $3.5 \mathrm{mg} / \mathrm{kg}$ (the CC7 group).

EDSS score was evaluated in terms of stability, improvement, or worsening each year, from CLARITY baseline over 5 years of follow-up (including a variable bridging interval between studies).
EDSS score remained stable for up to 5 years post-CLARITY baseline in over $50 \%$ of patients treated with cladribine tablets; the effects were similar in both patients receiving no further treatment or addition courses of cladribine tablets in years 3 and 4 of CLARITY Extension.

The analysis is consistent with long-term beneficial effects on disability afforded by treatment with cladribine tablets.

\section{INTRODUCTION}

Treatment objectives for patients with relapsing multiple sclerosis (RMS) include reducing risk of attacks (relapses) and delaying accrual of neurological impairment [1]. However, it is not clear how the efficacy observed in randomized clinical trials for disease-modifying therapies (DMTs) translates into longer-term treatment benefits in terms of effects on disability [2, 3]. Confirmed disability worsening, as assessed by the Expanded Disability Status Scale (EDSS), is an important endpoint in clinical trials of DMTs [4-6]. The EDSS ranges from 0 (normal neurological status) to 10 [death from multiple sclerosis (MS)] [7, 8], and can be used to monitor the time course and rate of worsening disability in such patients [9-11].

The two-year Cladribine Tablets Treating Multiple Sclerosis Orally (CLARITY) study demonstrated that, in patients with RMS, cladribine tablets $3.5 \mathrm{mg} / \mathrm{kg}$ significantly reduced relapse rates and improved findings on magnetic resonance imaging versus placebo [12]. Thereafter, the CLARITY Extension study concluded that treatment with cladribine tablets for 2 years followed by treatment with placebo for 2 years produced similar clinical benefits to 4 years of cladribine tablets treatment, but with a lower incidence of grade $3 / 4$ lymphopenia [13].

In this exploratory post hoc analysis we further evaluated the long-term effects on disability, assessed by the EDSS score, in patients with 
RMS who received cladribine tablets $3.5 \mathrm{mg} / \mathrm{kg}$ in the CLARITY [12] and CLARITY Extension [13] studies. Our aim was to answer an important clinical question-at the recommended cumulative dose of $3.5 \mathrm{mg} / \mathrm{kg}$ over 2 years (administered as two annual courses, each of 2 weeks and administered 1 month apart), what is the long-term effect of cladribine tablets on disability over 4 years or more? To do so, we evaluated patients who received cladribine tablets $3.5 \mathrm{mg} / \mathrm{kg}$ only during CLARITY [12] and those who received cladribine tablets $3.5 \mathrm{mg} / \mathrm{kg}$ during both CLARITY [12] and CLARITY Extension [13].

\section{METHODS}

The design and primary results of the CLARITY (NCT00213135) and CLARITY Extension (NCT00641537) studies, including efficacy and safety, have been reported previously [12-14]. Briefly, patients with RMS who were on placebo in CLARITY were assigned to cladribine tablets $3.5 \mathrm{mg} / \mathrm{kg}$ in CLARITY Extension (with blind maintained) while patients receiving any dose of cladribine tablets in CLARITY were re-randomized (2:1) to cladribine $3.5 \mathrm{mg} / \mathrm{kg}$ or placebo in the extension phase.

Patients enrolled into CLARITY Extension who were previously randomized to cladribine tablets $3.5 \mathrm{mg} / \mathrm{kg}$ in CLARITY were included in this post hoc analysis. Two treatment groups were investigated-patients randomized to cladribine tablets $3.5 \mathrm{mg} / \mathrm{kg}$ in CLARITY and thereafter randomized to placebo in CLARITY Extension (hereafter referred to as the CP3.5 group) or to cladribine tablets $3.5 \mathrm{mg} / \mathrm{kg}$ in CLARITY Extension (referred to as the CC7 group; Fig. 1). Given the 2:1 re-randomization for CLARITY Extension after 1:1:1 randomization schedule for CLARITY, there was an imbalance in group sizes for the CP3.5 and CC7 groups. Patients enrolled in CLARITY Extension who received higher doses of cladribine tablets $(5.25 \mathrm{mg} / \mathrm{kg})$ or placebo in CLARITY were not included in this analysis, in view of the research question outlined above.

For logistical reasons, there was a variable bridging interval before patients entered the extension phase after completing CLARITY; no cladribine tablets were administered during this interval. Among the overall study population, the length of time between completion of CLARITY and entry to CLARITY Extension varied from 0.1 to 118 weeks (median, 40.3 weeks) [13].

In CLARITY and CLARITY Extension, neurological examinations were conducted, and EDSS score determined, during the pre-study evaluation, on the first day of the study, and at weeks $13,24,36,48,60,72,84$, and 96 of the corresponding double-blind treatment periods. No EDSS scores were retrospectively collected for the bridging interval between CLARITY and CLARITY Extension. Patients were permitted to receive interferon beta or glatiramer acetate during the bridging interval, but discontinued any DMT at least 3 months before the first study day of CLARITY Extension.

\section{Endpoints}

We undertook two different and complementary approaches to analysing EDSS changes over five chronological years from CLARITY baseline: an unconfirmed approach which compared the minimum EDSS score in each year with the baseline EDSS score, and a confirmed EDSS progression approach using time to either 3- or 6-month confirmed EDSS progression. In the unconfirmed approach, EDSS score improvement or worsening was defined as any decrease or increase in minimum score in each year, respectively, depending on EDSS at baseline (at least 1.5 points, if baseline EDSS score $=0$; at least 1 point, if baseline EDSS score $\leq 4.5$; and at least 0.5 points, if baseline EDSS score $\geq 5$ ). All other cases were classified as stable.

\section{Compliance with Ethics Guidelines}

The CLARITY and CLARITY Extension studies were approved by local ethical review boards and were conducted in accordance with the principles of Good Clinical Practice and the Declaration of Helsinki. All patients provided written informed consent to participate. 


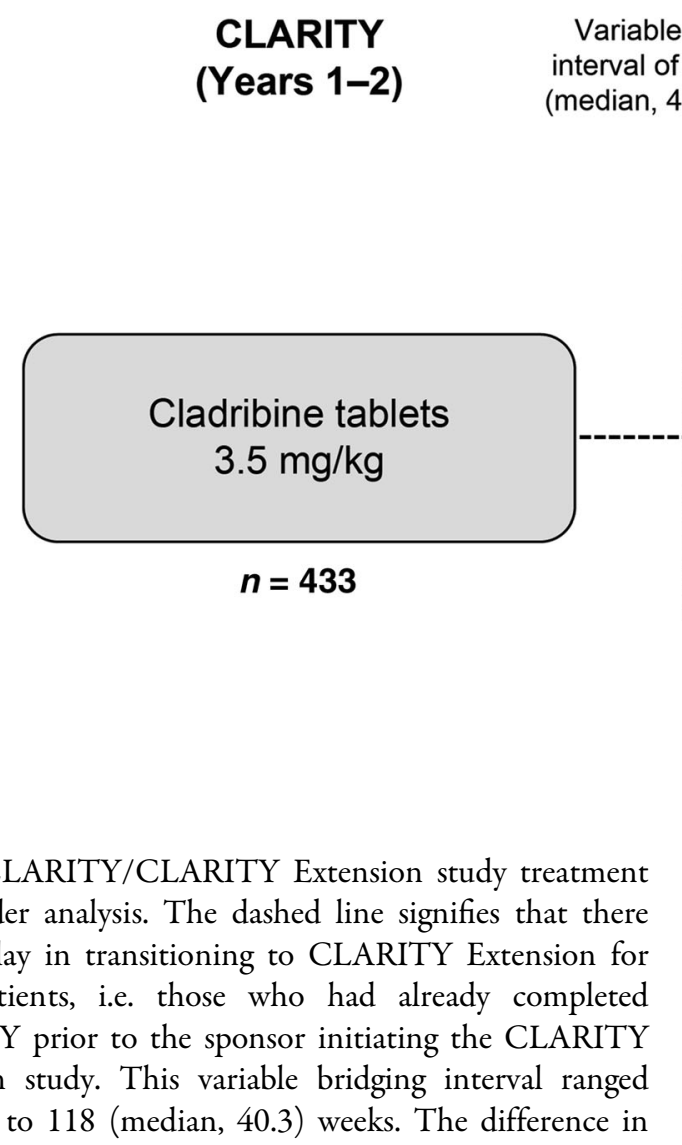

Fig. 1 CLARITY/CLARITY Extension study treatment arms under analysis. The dashed line signifies that there was a delay in transitioning to CLARITY Extension for some patients, i.e. those who had already completed CLARITY prior to the sponsor initiating the CLARITY Extension study. This variable bridging interval ranged from 0.1 to 118 (median, 40.3) weeks. The difference in

\section{Statistical Analysis}

This was an exploratory post hoc analysis in which EDSS score over time was descriptively analysed (median with 95\% confidence interval [CI], mean, interquartile range, minimum, and maximum) at 6-month intervals. The proportion of patients with improvement, worsening, and stability of EDSS score over 12 months is also presented $(n, \%)$. Three- and 6-month confirmed EDSS progression from CLARITY entry are presented by means of Kaplan-Meier plots. As a result of the uncontrolled bridging period and the 2:1 re-randomization at the beginning of CLARITY Extension, no inferential statistical analyses were performed to compare treatment groups.

\section{CLARITY Extension (Years 3-4)}

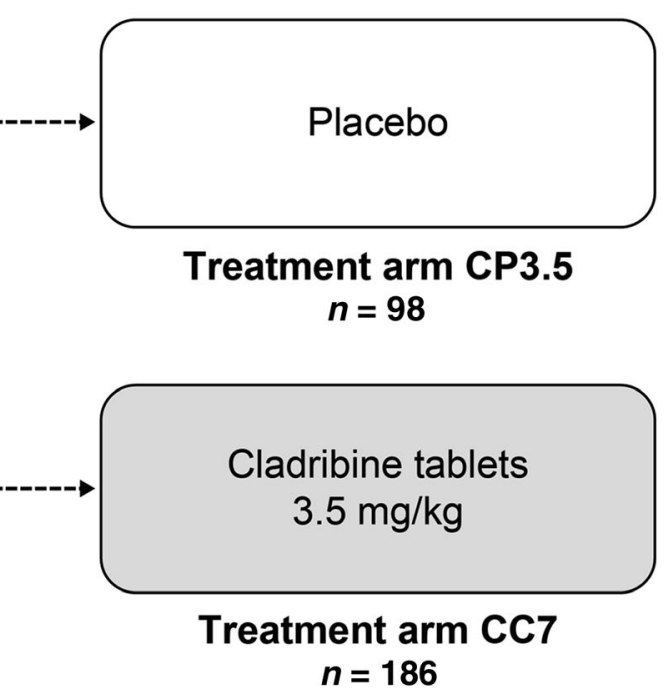

group sizes is an artefact of the re-randomization schedule, in that patients who received cladribine tablets in CLARITY were re-randomized $(2: 1)$ to cladribine tablets $3.5 \mathrm{mg} / \mathrm{kg}$ or placebo in CLARITY Extension. Consequently, the CC7 group contained twice as many patients as the CP3.5 group

\section{RESULTS}

Of the 1326 patients in CLARITY, 806 (60.8\%) patients followed on into CLARITY Extension. Demographics and clinical characteristics at baseline of CLARITY and CLARITY Extension for patients in the CP3.5 group $(n=98)$ and the CC7 group $(n=186)$ are shown in Table 1 . No patients in either group received additional DMTs in the 3 months before randomization to treatment in CLARITY Extension, and only two patients, both in the CP3.5 group, received DMTs at any time in the bridging interval between studies. Another DMT was started not because of worsening but because the patient or physician felt that it was best that the patient was receiving a DMT during this time. Demographics and clinical characteristics (including median EDSS at baseline) were comparable between groups, and the median intervals 
Table 1 Characteristics of patients included in the analysis at baseline of CLARITY and CLARITY Extension

\begin{tabular}{|c|c|c|c|c|}
\hline \multirow[t]{2}{*}{ Parameter } & \multicolumn{2}{|c|}{ CP3.5 $(n=98)$} & \multicolumn{2}{|c|}{ CC7 $(n=186)$} \\
\hline & $\begin{array}{l}\text { CLARITY } \\
\text { baseline }\end{array}$ & $\begin{array}{l}\text { CLARITY } \\
\text { Extension } \\
\text { baseline }\end{array}$ & $\begin{array}{l}\text { CLARITY } \\
\text { baseline }\end{array}$ & $\begin{array}{l}\text { CLARITY } \\
\text { Extension } \\
\text { baseline }\end{array}$ \\
\hline Mean age, years (SD) & $38.1(10.6)$ & $40.7(10.7)$ & $37.9(10.4)$ & $40.6(10.5)$ \\
\hline $\begin{array}{l}\text { Previously used DMT before randomization in } \\
\text { CLARITY, } n(\%)\end{array}$ & $18(18.4)$ & - & $43(23.1)$ & - \\
\hline DMT use between CLARITY and Extension, $n$ (\%) & - & $2(2.0)$ & - & 0 \\
\hline $\begin{array}{l}\text { Prior DMT within } 3 \text { months of Extension study } \\
\text { day } 1, n(\%)\end{array}$ & - & 0 & - & 0 \\
\hline \multicolumn{5}{|l|}{ Relapses in 12 months prior to CLARITY, $n$ (\%) } \\
\hline 0 & $0(0)$ & - & $0(0)$ & - \\
\hline 1 & $69(70.4)$ & - & $135(72.6)$ & - \\
\hline 2 & $20(20.4)$ & - & $44(23.7)$ & - \\
\hline$\geq 3$ & $9(9.2)$ & - & $7(3.8)$ & - \\
\hline $\begin{array}{l}\text { Patients reporting at least one relapse between } \\
\text { CLARITY and Extension, }{ }^{a} n(\%)\end{array}$ & - & $9(9.2)$ & - & $17(9.1)$ \\
\hline \multicolumn{5}{|l|}{ EDSS at baseline } \\
\hline Median & 3.0 & 2.5 & 2.5 & 2.5 \\
\hline Interquartile range & $2.0-4.0$ & $2.0-4.0$ & $2.0-3.5$ & $1.5-4.0$ \\
\hline Min; $\max$ & $0.0 ; 5.5$ & $0.0 ; 6.5$ & $0.0 ; 6.0$ & $0.0 ; 6.5$ \\
\hline
\end{tabular}

67 (68.4\%) patients in the CP3.5 group and 124 (66.7\%) in the CC7 group were female

CC7 patients randomized to cladribine tablets $3.5 \mathrm{mg} / \mathrm{kg}$ in both CLARITY and CLARITY Extension, CP3.5 patients randomized to cladribine tablets $3.5 \mathrm{mg} / \mathrm{kg}$ in CLARITY and placebo in CLARITY Extension, DMT disease-modifying therapy, EDSS Expanded Disability Status Scale, $S D$ standard deviation

${ }^{a}$ Relapses during the bridging interval were not qualified by an evaluating physician

between studies were similar (41.3 weeks for the CP3.5 group and 41.4 weeks for the CC7 group).

\section{EDSS Over Time}

EDSS scores at 6-month intervals over 5 years in each treatment group are shown in Fig. 2. Five years after CLARITY baseline, including the variable bridging interval, median EDSS score remained stable compared with baseline values for both the CP3.5 and CC7 groups. The median EDSS score remained between 2.0 and 3.0 up to
5 years in the CP3.5 group; mean [standard deviation (SD)] EDSS score at 5 years was 3.05 (1.64) and mean change from CLARITY baseline was 0.03 (0.98). In the CC7 group, median EDSS score ranged between 2.0 and 2.5 up to 5 years; mean (SD) EDSS at this time point was 2.63 (1.5), and mean change from CLARITY baseline was $0.08(0.98)$.

The median (95\% CI) EDSS score for patients in the CP3.5 group at year $5(n=69)$ was 2.5 (2.0-3.5) compared with $3.0(2.5-3.5)$ at baseline. In the CC7 group, median EDSS score (95\% 


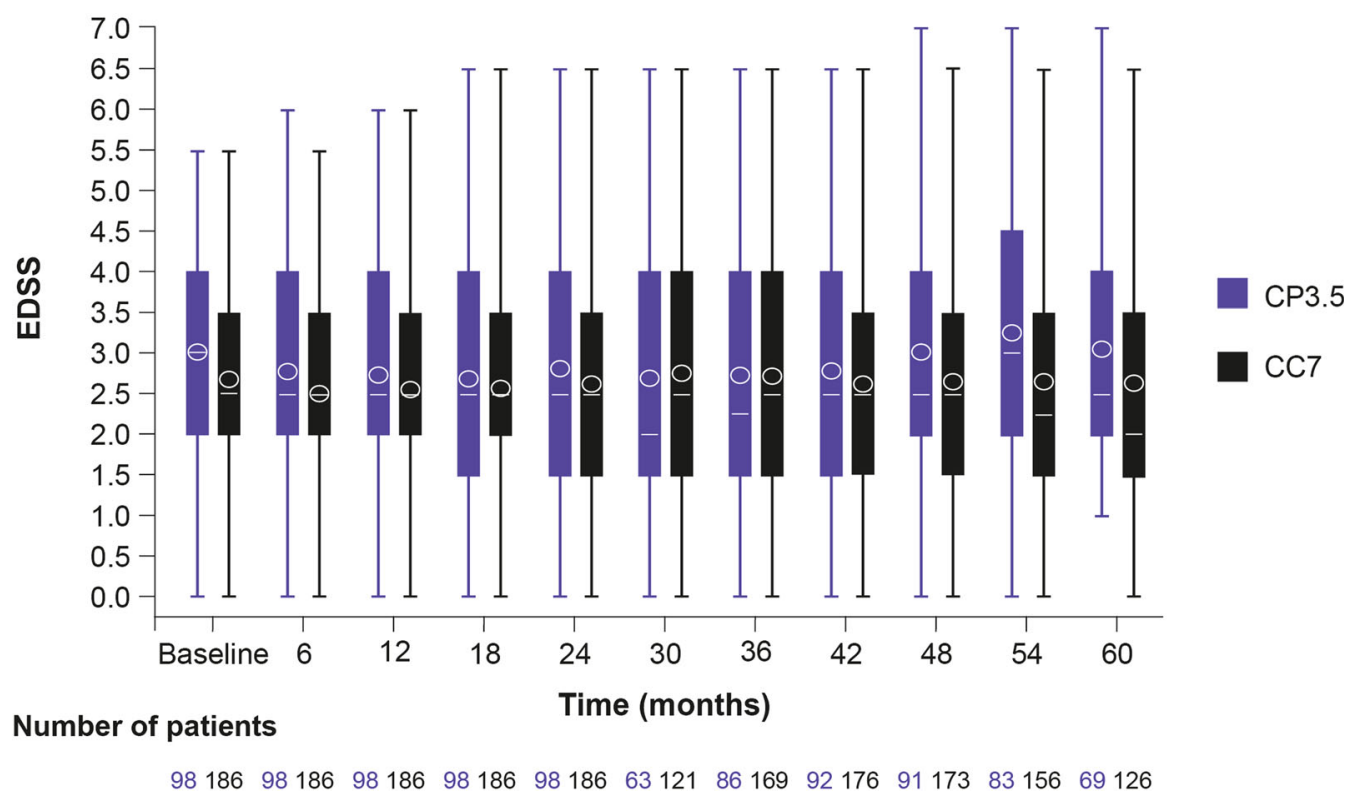

Fig. 2 EDSS scores over time in the CP3.5 and CC7 patient groups. Line $=$ Median, Circles $=$ Mean, Box $=$ Q1, Q3. Lower and upper whiskers reflect the minimum and maximum EDSS score. CC7, patients randomized to cladribine tablets $3.5 \mathrm{mg} / \mathrm{kg}$ in both CLARITY and

CI) at year $5(n=126)$ was $2.0(2.0-3.0)$ compared with 2.5 (2.5-3.0) at baseline.

\section{Annual EDSS Stability}

In the CP3.5 group, in each yearly period, EDSS score was stable in $53.9-76.5 \%$ of patients, improved in 21.3-29.6\%, and worsened in 0-24.7\% (Fig. 3). In the CC7 group, in each yearly period, EDSS score was stable in $65.4-81.7 \%$ of patients, improved in 16.7-20.3\%, and worsened in 1.6-15.8\%.

During year 5, EDSS score stability was observed in 48 (53.9\%) patients, improvement in $19(21.3 \%)$, and worsening in $22(24.7 \%)$ in the CP3.5 group $(n=89)$. In the corresponding CC7 group during year $5(n=171)$, EDSS score remained stable in 113 patients (66.1\%), improved in $31(18.1 \%)$, and worsened in 27 (15.8\%).
CLARITY Extension; CP3.5, patients randomized to cladribine tablets $3.5 \mathrm{mg} / \mathrm{kg}$ in CLARITY and placebo in CLARITY Extension; EDSS, Expanded Disability Status Scale. Reprinted with permission from ePresentation Sessions. Eur J Neurol 2020;27(Suppl. 1):468

\section{Time to 3-month or 6-month Confirmed EDSS Progression}

In both the CP3.5 and CC7 groups, less than $31 \%$ and $27 \%$ of patients, respectively, reached 3-month confirmed EDSS progression by year 5 (Fig. 4). A similar pattern was observed with time to 6-month confirmed EDSS progression, with less than $30 \%$ of patients having confirmed 6-month confirmed EDSS progression by year 5 in both the CP3.5 and CC7 groups (Fig. 4).

\section{DISCUSSION}

With the present availability of a large number of DMTs for RMS, efficacy, particularly the ability of DMTs to reduce the risk of relapses and disability worsening [15-17], has a key role in treatment choices. Disability accumulation in MS may occur as a progression independent of relapses activity (PIRA) or in association with relapses $[18,19]$. Analyses such as that reported 


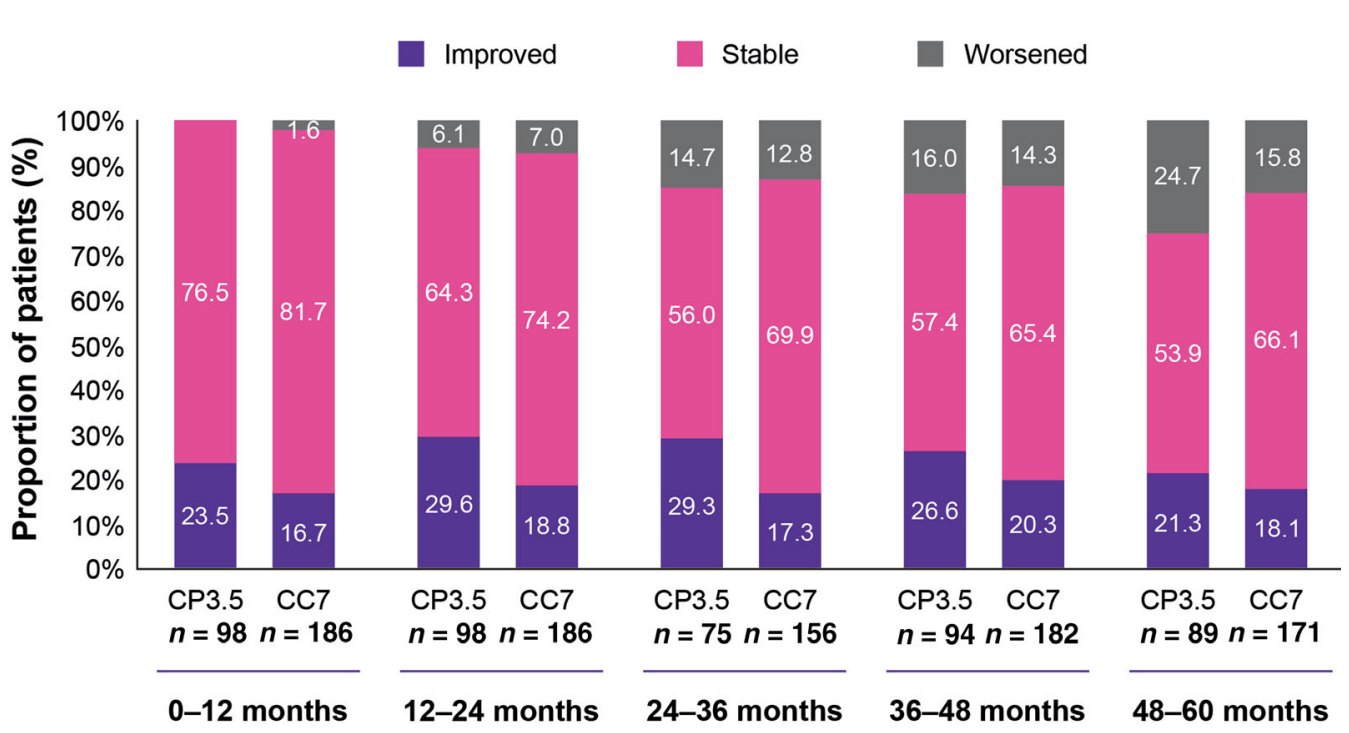

Fig. 3 Change in EDSS score in each 12-month period up to 5 years in the CP3.5 and CC7 patient groups. Improvement, worsening, and stability of EDSS score over 12 months were descriptively analysed using the minimum EDSS score for each 12-month period. CC7, patients randomized to cladribine tablets $3.5 \mathrm{mg} / \mathrm{kg}$ in both

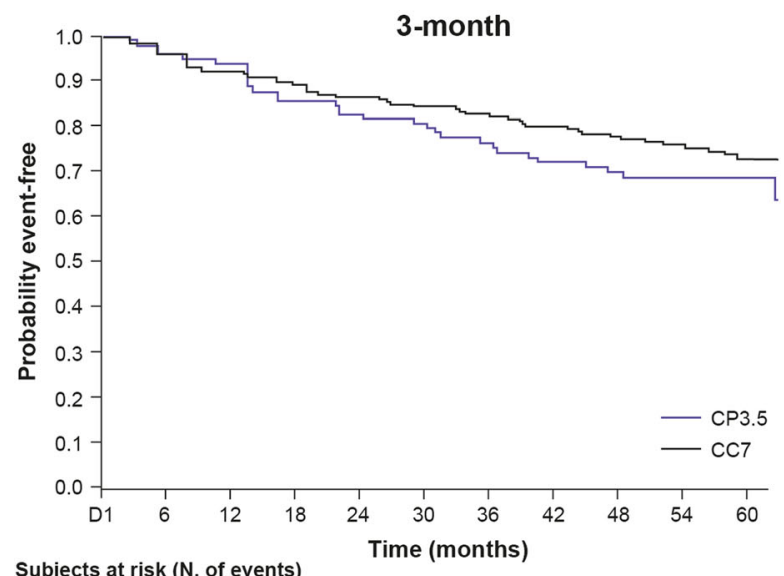

Subjects at risk (N. of events)

$\begin{array}{llllllllllll}\text { CP3.5 } & 98(0) & 94(4) & 92(6) & 84(14) & 81(17) & 78(19) & 73(23) & 68(27) & 63(29) & 58(30) & 39(30)\end{array}$ $\begin{array}{llllllllllll}\text { CC7 } & 186(0) & 179(7) & 172(14) & 166(20) & 161(25) & 157(29) & 152(32) & 145(37) & 136(41) & 115(44) & 70(48)\end{array}$

Fig. 4 Time to 3- and 6-month confirmed EDSS worsening from CLARITY entry in the CP3.5 and CC7 patient groups. CC7, patients randomized to cladribine tablets $3.5 \mathrm{mg} / \mathrm{kg}$ in both CLARITY and CLARITY

here can therefore provide additional information that is relevant to both patients and physicians when considering treatment options, including the question of the length of
CLARITY and CLARITY Extension; CP3.5, patients randomized to cladribine tablets $3.5 \mathrm{mg} / \mathrm{kg}$ in CLARITY and placebo in CLARITY Extension; EDSS, Expanded Disability Status Scale. Reprinted with permission from ePresentation Sessions. Eur J Neurol 2020;27(Suppl. 1):468

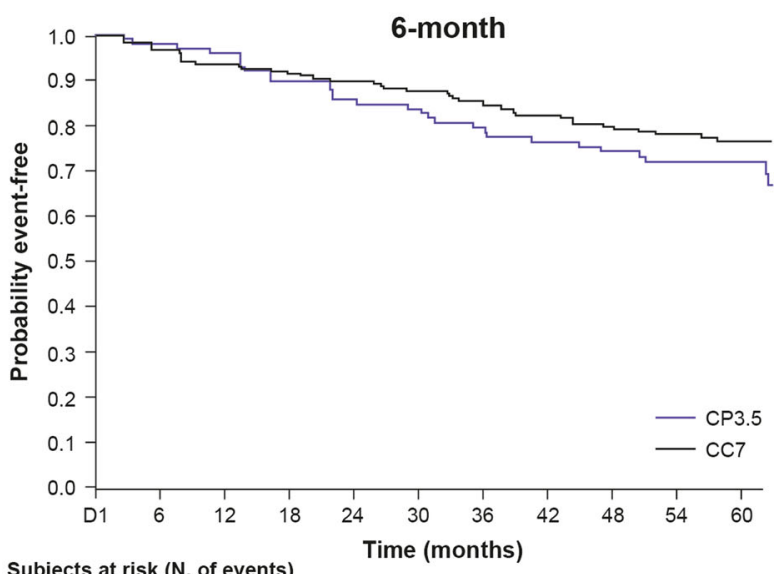

Subjects at risk (N. of events)

$\begin{array}{llllllllllll}\mathrm{CP} 3.5 & 98(0) & 96(2) & 94(4) & 88(10) & 84(14) & 81(16) & 76(20) & 72(23) & 66(25) & 59(27) & 40(27)\end{array}$ CC7 $186(0) \quad 180(6) \quad 174(12) 170(16) 167(19) \quad 163(23) 157(27) \quad 149(33) 139(37) \quad 116(40) \quad 72(42)$

Extension; CP3.5, patients randomized to cladribine tablets $3.5 \mathrm{mg} / \mathrm{kg}$ in CLARITY and placebo in CLARITY Extension; EDSS, Expanded Disability Status Scale

time before patients may require additional treatment. This is particularly true for DMTs with a pronounced and long-lasting effect on the immune system. Indeed, a clear 
understanding of treatment benefits and risks by the patient is fundamental to shared decision-making, and it has been reported that this understanding can be improved by appropriate communication of treatment effects, in particular describing anticipated changes in absolute terms in conjunction with baseline information [17].

Avoiding a cumulative increase in patient disability is a key aim of treatment for MS [1]. Information on the proportion of patients with stabilization of disability (and improvement, if possible) at time periods from initiation of treatment, as reported here, may therefore be valuable for physicians to discuss with patients. This post hoc analysis suggests that for up to 5 years after initiation of treatment with cladribine tablets, approximately two out of three patients had a stable or improved EDSS score (in contrast, in untreated patients, EDSS score would be expected to increase over this time [10]). Of particular note is that there were few differences in the proportion of patients with stable EDSS score out to year 5 between patients treated with cladribine tablets only in years 1 and 2 (CP3.5 group) and those who received four annual courses (CC7 group). Such findings confirm the long-term effect of cladribine tablets on disability at the recommended dose of $3.5 \mathrm{mg} / \mathrm{kg}$ over 2 years, without the need for additional treatment in years 3 and 4. Notably, less than $31 \%$ of patients treated with the recommended dose of $3.5 \mathrm{mg} / \mathrm{kg}$ over 2 years had confirmed EDSS progression by the end of year 5 .

A key question with the use of proposed immune reconstitution therapies such as cladribine tablets is how to monitor and provide further treatment to patients, if required, after the initial course of treatment. While there was little difference in EDSS outcomes in patients receiving two courses of cladribine tablets versus those receiving four courses, it is important to consider that some differences were observed in neurological endpoints in CLARITY Extension. These differences were primarily due to $\mathrm{T} 1$ gadolinium-enhancing lesions occurring in a small subset of patients who were randomized to placebo after two courses of cladribine tablets and in those patients with the maximum time since the CLARITY study. An expert opinion using Delphi methodology has suggested that patients with new or reappearing disease activity after year 4 could consider either additional courses of cladribine tablets or a switch to another high-efficacy therapy, following discussion with their physician [20].

Increasing evidence suggests that treatment with cladribine tablets is associated with a longterm reduction of memory $B$ cells that persists after overall lymphocyte counts have recovered from the initial reduction, which occurs 2-3 months after the beginning of each treatment course [21-23]. There appears to be no change in the risk of infections outside of the periods of when total lymphocyte count is reduced, suggesting that the sustained clinical effect is not associated with the potential risks associated with immunosuppression [13, 24].

Some caveats and limitations to this exploratory analysis should be acknowledged. First, comparison of the two treatment groups was not an objective of the analysis. Second, differences in the size of the two groups introduced by 2:1 re-randomization in the extension study were limitations to interpretation of results. Timing of re-randomization was not standardized because of the variable bridging interval, and perception of patients at time of re-randomization can influence reporting. Third, data at 5-year follow-up were only available for $70 \%(69 / 98)$ of patients in the CP3.5 group and 68\% (126/186) in the CC7 group; as such, data for up to approximately $30 \%$ of patients whose EDSS score is shown for CLARITY are not represented in the values at the end of CLARITY Extension. However, loss to follow-up and missing data are common challenges in the extension phases of clinical trials. We used the lowest (minimum) EDSS score obtained in any year of the study for the purposes of comparing EDSS score to baseline. An alternative approach would be to use the highest EDSS value in each year. However, while EDSS scores assessed within 30 days of relapse onset were excluded from the current analysis, the longer-term effect of relapses on EDSS could not be excluded. As such, findings for 3- or 6-month confirmed EDSS progression were also explored. The effect of treatments used in the bridging 
interval between CLARITY and CLARITY Extension were not analysed. However, only two patients in the CP3.5 group received DMTs at any time in the bridging interval, and no patients in either group received additional DMTs in the 3 months before randomization to treatment in CLARITY Extension, so this is unlikely to have had an effect on the outcomes observed. Finally, for patients re-randomized to cladribine tablets $3.5 \mathrm{mg} / \mathrm{kg}$ in CLARITY Extension, the period of follow-up exposed to the treatment was quite variable because of the variability of the bridging interval.

\section{CONCLUSIONS}

EDSS score remained stable for up to 5 years post-CLARITY baseline in over $50 \%$ of patients treated with cladribine tablets. The effects were similar in both patients receiving no further treatment or addition courses of cladribine tablets in years 3 and 4 of CLARITY Extension. Over $70 \%$ of patients did not show any confirmed disability worsening during the entire follow-up, and a similar value was also observed in patients who received treatment with cladribine tablets during the CLARITY study only. These findings attest to the long-term disease stability afforded by either the recommended cumulative dose of cladribine tablets over 4 years $(3.5 \mathrm{mg} / \mathrm{kg})$ or a higher cumulative dose, using a disability endpoint (EDSS) of particular importance to patients.

\section{ACKNOWLEDGEMENTS}

The authors would like to thank patients and their families, investigators, co-investigators, and the study teams at each of the participating centres and at the healthcare business of Merck KGaA, Darmstadt, Germany.

Funding. The healthcare business of Merck KGaA, Darmstadt, Germany (CrossRef Funder ID: https://doi.org/10.13039/100009945) provided funding for this analysis and payment of Rapid Service and Open Access Fees.
Medical Writing Assistance. Medical writing assistance for the development of this manuscript, under the direction of the authors, was provided by Matthew Bexon, Joseph Ward, and Steve Winter of inScience Communications, Springer Healthcare Ltd, UK, and was funded by the healthcare business of Merck KGaA, Darmstadt, Germany.

Authorship. All named authors meet the International Committee of Medical Journal Editors (ICMJE) criteria for authorship for this article and take responsibility for the integrity of the work as a whole.

Authors' Contributions. Conceptualization, investigation, data acquisition and interpretation: Gavin Giovannoni, Giancarlo Comi, Kottil Rammohan, Peter Rieckmann, and Patrick Vermersch. Conceptualization and data interpretation: Fernando Dangond and Dominic Jack. Conceptualization, formal analysis, and data interpretation: Birgit Keller. All authors contributed to writing of the manuscript and have given their approval for this version to be published.

Prior Presentation. The findings summarized in this publication were previously presented at the virtual congress of the European Association of Neurology (23-26 May 2020).

Disclosures. Gavin Giovannoni has received speaker honoraria and consulting fees from AbbVie, Actelion (Janssen/J\&J), Atara Bio, Almirall, Bayer, Biogen, Celgene (BMS), FivePrime, GlaxoSmithKline, GW Pharma, Ironwood, the healthcare business of Merck KGaA (Darmstadt, Germany), Novartis, Merck \& Co., Pfizer Inc., Protein Discovery Laboratories, Roche, Sanofi-Genzyme, Teva Pharmaceutical Industries Ltd, UCB, and Vertex Pharmaceuticals; and has received research support unrelated to this study from Biogen, Ironwood, the healthcare business of Merck KGaA (Darmstadt, Germany), Novartis, Merck \& Co., and Takeda. Giancarlo Comi has received consulting fees from Bayer, Biogen, the healthcare business of Merck KGaA (Darmstadt, Germany), Novartis, Receptos, Roche/Genentech, Sanofi-Aventis, 
and Teva Pharmaceutical Industries Ltd; lecture fees from Bayer, Biogen, the healthcare business of Merck KGaA (Darmstadt, Germany), Novartis, Sanofi-Aventis, Serono Symposia International Foundation, and Teva Pharmaceutical Industries Ltd; and trial grant support from Bayer, Biogen, the healthcare business of Merck KGaA (Darmstadt, Germany), Novartis, Receptos, Roche/Genentech, Sanofi-Aventis, and Teva Pharmaceutical Industries Ltd. Kottil Rammohan has received honoraria for lectures and steering committee meetings from Acorda, Biogen, EMD Serono, Genzyme, Novartis, Roche/Genentech, Sanofi-Aventis, and Teva Neuroscience. Peter Rieckmann has received honoraria for lectures/steering committee meetings from Bayer, Boehringer Ingelheim, Biogen, Genzyme, the healthcare business of Merck KGaA (Darmstadt, Germany), Novartis, Sanofi-Aventis, and Teva Pharmaceutical Industries, and Serono Symposia International Foundation. Fernando Dangond is an employee of EMD Serono Research \& Development Institute, Inc., Billerica, MA, USA. Birgit Keller is an employee of the healthcare business of Merck KGaA, Darmstadt, Germany. Dominic Jack is an employee of Merck Serono Ltd, Feltham, UK (an affiliate of Merck KGaA, Darmstadt, Germany). Patrick Vermersch has received honoraria or consulting fees from Biogen, Celgene (BMS), Imcyse, the healthcare business of Merck KGaA (Darmstadt, Germany), Novartis, Roche, SanofiGenzyme, and Teva; and has received research support from Novartis, Roche, and SanofiGenzyme.

Compliance with Ethics Guidelines. The CLARITY and CLARITY Extension studies were approved by local ethical review boards and were conducted in accordance with the principles of Good Clinical Practice and the Declaration of Helsinki. All patients provided written informed consent to participate.

Data Availability. Any requests for data by qualified scientific and medical researchers for legitimate research purposes will be subject to the healthcare business of Merck KGaA's Data Sharing Policy. All requests should be submitted in writing to the healthcare business of Merck
KGaA's data sharing portal https://www. merckgroup.com/en/research/our-approach-toresearch-and-development/healthcare/clinicaltrials/commitment-responsible-data-sharing. html. When the healthcare business of Merck $\mathrm{KGaA}$ has a co-research, co-development, or comarketing or co-promotion agreement, or when the product has been out-licensed, the responsibility for disclosure might be dependent on the agreement between parties. Under these circumstances, the healthcare business Merck $\mathrm{KGaA}$ will endeavor to gain agreement to share data in response to requests.

Open Access. This article is licensed under a Creative Commons Attribution-NonCommercial 4.0 International License, which permits any non-commercial use, sharing, adaptation, distribution and reproduction in any medium or format, as long as you give appropriate credit to the original author(s) and the source, provide a link to the Creative Commons licence, and indicate if changes were made. The images or other third party material in this article are included in the article's Creative Commons licence, unless indicated otherwise in a credit line to the material. If material is not included in the article's Creative Commons licence and your intended use is not permitted by statutory regulation or exceeds the permitted use, you will need to obtain permission directly from the copyright holder. To view a copy of this licence, visit http://creativecommons.org/licenses/bync/4.0/.

\section{REFERENCES}

1. Thompson AJ, Baranzini SE, Geurts J, Hemmer B, Ciccarelli O. Multiple sclerosis. Lancet. 2018;391(10130):1622-36.

2. Capra R, Cordioli C, Rasia S, et al. Assessing longterm prognosis improvement as a consequence of treatment pattern changes in MS. Mult Scler. 2017;23(13):1757-61.

3. Giovannoni G. Do we have equipoise when it comes to how we treat active multiple sclerosis? Lancet Neurol. 2019;18(10):909-11. 
4. Wiendl H, Meuth SG. Pharmacological approaches to delaying disability progression in patients with multiple sclerosis. Drugs. 2015;75(9):947-77.

5. Cohen JA, Reingold SC, Polman CH, Wolinsky JS. Disability outcome measures in multiple sclerosis clinical trials: current status and future prospects. Lancet Neurol. 2012;11(5):467-76.

6. D'Souza M, Heikkilä A, Lorscheider J, et al. Electronic Neurostatus-EDSS increases the quality of Expanded Disability Status Scale assessments: experience from two phase 3 clinical trials. Mult Scler. 2020;26(8):993-6.

7. Kurtzke JF. Rating neurologic impairment in multiple sclerosis: an Expanded Disability Status Scale (EDSS). Neurology. 1983;33(11):1444-52.

8. Kurtzke JF. Historical and clinical perspectives of the Expanded Disability Status Scale. Neuroepidemiology. 2008;31(1):1-9.

9. Weinshenker BG, Bass B, Rice GP, et al. The natural history of multiple sclerosis: a geographically based study. I. Clinical course and disability. Brain. 1989;112(Pt 1):133-46.

10. Confavreux C, Vukusic S. The clinical course of multiple sclerosis. Handb Clin Neurol. 2014;122: 343-69.

11. Brown MG, Asbridge M, Hicks V, et al. Estimating typical multiple sclerosis disability progression speed from clinical observations. PLoS ONE. 2014;9(10): e105123.

12. Giovannoni G, Comi G, Cook S, et al. A placebocontrolled trial of oral cladribine for relapsing multiple sclerosis. N Engl J Med. 2010;362(5): 416-26.

13. Giovannoni G, Soelberg Sørensen P, Cook S, et al. Safety and efficacy of cladribine tablets in patients with relapsing-remitting multiple sclerosis: results from the randomized extension trial of the CLARITY study. Mult Scler. 2018;24(12):1594-604.

14. Comi G, Cook S, Rammohan K, et al. Long-term effects of cladribine tablets on MRI activity outcomes in patients with relapsing-remitting multiple sclerosis: the CLARITY Extension study. Ther Adv Neurol Disord. 2018;11: 1756285617753365.
15. Brown H, Gabriele S, White J. Physician and patient treatment decision-making in relapsing-remitting multiple sclerosis in Europe and the USA. Neurodegener Dis Manag. 2018;8(6):371-6.

16. Eskyte I, Manzano A, Pepper G, et al. Understanding treatment decisions from the perspective of people with relapsing remitting multiple Sclerosis: a critical interpretive synthesis. Mult Scler Relat Disord. $2019 ; 27: 370-7$.

17. Reen GK, Silber E, Langdon DW. Best methods of communicating clinical trial data to improve understanding of treatments for patients with multiple sclerosis. Value Health. 2018;21(7):762-6.

18. Bsteh $G$, Hegen $H$, Altmann $P$, et al. Retinal layer thinning is reflecting disability progression independent of relapse activity in multiple sclerosis. Mult Scler J Exp Transl Clin. 2020;6(4): 2055217320966344.

19. Kappos L, Wolinsky JS, Giovannoni G, et al. Contribution of relapse-independent progression vs relapse-associated worsening to overall confirmed disability accumulation in typical relapsing multiple sclerosis in a pooled analysis of 2 randomized clinical trials. JAMA Neurol. 2020;77(9):1132-40.

20. Soelberg Sørensen P, Centonze D, Giovannoni G, et al. Expert opinion on the use of cladribine tablets in clinical practice. Ther Adv Neurol Disord. 2020;13:1-17.

21. Comi G, Cook S, Giovannoni G, et al. Effect of cladribine tablets on lymphocyte reduction and repopulation dynamics in patients with relapsing multiple sclerosis. Mult Scler Relat Disord. 2019;29: 168-74.

22. Stuve O, Soelberg Sørensen P, Leist T, et al. Effects of cladribine tablets on lymphocyte subsets in patients with multiple sclerosis: an extended analysis of surface markers. Ther Adv Neurol Disord. 2019;12: 1756286419854986.

23. Baker D, Pryce G, Herrod SS, Schmierer K. Potential mechanisms of action related to the efficacy and safety of cladribine. Mult Scler Relat Disord. 2019;30:176-86.

24. Cook S, Leist T, Comi G, et al. Safety of cladribine tablets in the treatment of patients with multiple sclerosis: an integrated analysis. Mult Scler Relat Disord. 2019;29:157-67. 